\title{
Glucose starvation as cancer treatment: Thermodynamic point of view
}

\author{
Durán $I^{1 *}$, Pomuceno-Orduñez ${ }^{1}$, Martin RR ${ }^{1,2}$, Silva E ${ }^{1}$, Montero $S^{1}$, Mansilla $R^{1,3}$, Cocho $G^{1,4}$ and Nieto-Villar JM ${ }^{1 *}$ \\ ${ }^{1}$ Department of Chemical-Physics, A. Alzola Group of Thermodynamics of Complex Systems M.V. Lomonosov Chemistry Chair, Faculty of Chemistry, University \\ of Havana, Cuba \\ ${ }^{2}$ Instituto de Investigaciones en Tecnología Química (INTEQUI), UNSL - CONICET, Argentina \\ ${ }^{3}$ Centro de Investigaciones Interdisciplinarias en Ciencias y Humanidades, UNAM, México \\ ${ }^{4}$ Instituto de Sistemas Complejos C3 \& Instituto de Física de la UNAM, México
}

\begin{abstract}
To evaluate the effect of glucose deprivation on the robustness of cancer glycolysis, the total entropy production rate was calculated from the in silico modeling of the glycolytic network of HeLa cells grown under different glucose and oxygen conditions. It was shown that glucose deprivation had a deleterious effect for the cells grown under hypoglycemia and hypoxia and that the intracellular acidification therapy and the glucose deprivation treatment had synergic effects on the decrease of cancer glycolysis robustness.
\end{abstract}

\section{Introduction}

Cancer, the second leading cause of death worldwide [1], is a generic name given to a complex interaction network of malignant cells that have lost their specialization and control over normal growth [2]. This behavior stems from to the accumulation of multiple DNA mutations in specific genes called oncogenes and tumor suppressor genes [3].

The design and development of new cancer drugs may take several years and in most cases will only be effective for a fraction of patients with specific types of cancer. Therefore, it is important to develop complementary strategies that can be quickly translated into effective therapies [4].

Cancer cells are characterized for maintaining a high rate of glycolysis, thus converting glucose to lactate at high speed, even in the presence of oxygen. This phenomenon is known as "aerobic glycolysis" or "Warburg effect". Numerous therapies against cancer are based on the inhibition of this metabolic network [5]. It is known that glucose deprivation has deleterious effects on cancer glycolysis which may even conduce to cell death [6].

Mutations and epigenetic modifications that increase growth and promote insensitivity to anti-growth signals in cancer cells, lead to the loss of appropriate responses to rapidly adapt to a variety of extreme environments including starvation [7]. Under challenging conditions such as starvation, normal cells reduce energy expenditure and divert it from growth to maintenance; thereby enhancing protection and survival [8]. However, the constitutive activation of oncoproteins can block entry into this protective mode in cancer cells; thus providing a method by which fasting induces protection in normal cells but not in oncogene-driven cancer cells, an effect called Differential Stress Resistance $[4,9]$.

Hypoxia arises in tumors through the uncontrolled oncogene driven proliferation of cancer cells in the absence of an efficient vascular bed. Due to the rapid proliferation of cancer cells, the tumor quickly exhausts the nutrient and oxygen supply from the normal vasculature, and becomes hypoxic [10].
There is a "metabolic symbiosis" between hypoxic and aerobic cancer cells inside a tumor, in which lactate produced by hypoxic cells is taken up by aerobic cells and it is used as their principal substrate for oxidative phosphorylation. As a result, the limited glucose available to the tumor is most efficiently used [11].

On the other hand, hypoxia correlates with therapeutic resistance to both cytotoxic drugs and radiotherapy [12]. The adaptation of tumor cells to hypoxia contributes to the malignant phenotype and to aggressive tumor progression [13]. Under hypoxia, the complexity and robustness of cancer glycolysis is higher than under normoxia [14].

Another hallmark of cancer cells is the reversed $\mathrm{pH}$ gradient: intracellular $\mathrm{pH}(\mathrm{pHi})$ are increased compared to normal cells $(\sim 7.3-$ 7.6 versus $\sim 7.2)$, while extracellular $\mathrm{pH}(\mathrm{pHe})$ is decreased $(\sim 6.8-7.0$ versus $\sim 7.4)$. The dysregulated $\mathrm{pH}$ of cancer cells enables cellular processes that are sensitive to small changes in $p H i$, including cell proliferation, migration and metabolism. These global cell biological effects are produced by the $\mathrm{pH}$-sensitive functions of proteins with activities or ligand-binding affinities that are regulated within the narrow cellular range of $\mathrm{pHi}$ dynamics [15].

The aim of this work is to evaluate the effect of glucose deprivation on cancer robustness through the entropy production rate $[16,17]$ of

*Correspondence to: Ileana Durán, Department of Chemical-Physics, A. Alzola Group of Thermodynamics of Complex Systems M.V. Lomonosov Chemistry Chair, Faculty of Chemistry, University of Havana, Havana 10400, Cuba, E-mail: ileduranadn@gmail.com

Nieto-Villar JM, Department of Chemical-Physics, A. Alzola Group of Thermodynamics of Complex Systems M.V. Lomonosov Chemistry Chair, Faculty of Chemistry, University of Havana, Havana 10400, Cuba, E-mail: nieto@fq.uh.cu

Received: May 02, 2018; Accepted: May 28, 2018; Published: May 31, 2018 
the glycolytic network for HeLa cells. The paper is organized as follows: In section 2.1, the kinetic models and the experimental procedure are described. In section 2.2 the thermodynamic formalism for the entropy production rate is presented. Section 3 focuses on the results obtained and section 4 comprehends the discussion of the results. Finally, some concluding remarks are exposed. The results achieved will be useful for the design of new cancer therapies based on glucose deprivation and for the understanding of their effect on the different solid tumor areas.

\section{Materials and methods}

\section{Kinetic model of cancer glycolysis}

The models used were proposed by Marin et al. [18] for the glycolytic network of HeLa tumor cell lines grown under three metabolic states during 24 hours, the sufficient time to induce phenotypic changes in cellular metabolism: Hypoglycemia (2.5 mM), Normoglycemia ( $5 \mathrm{mM})$ and Hyperglycemia (25 mM), all the three under normoxia. However, the growth saturation was not attained within this time but in a second phase where the cells were exposed to different glucose concentrations: $2.5 \mathrm{mM}, 5 \mathrm{mM}$ and $25 \mathrm{mM}$, until they reached the stationary state [18].

An intermittent fasting therapy was simulated with the $5 \mathrm{mM}$ second phase models, for the three metabolic phenotypes, as the one carried out in the study of Lee et al. [7]. Heaviside Step Function [19] was used to perturb the extracellular glucose concentration $\left(\mathrm{Glc}_{\text {out }}\right)$ by switching it from a fasting state $(1 \mathrm{mM})$ to a normoglycemic state (5 $\mathrm{mM}$ ) with 24 and 12 hours' periods for the first and second experiment respectively. In such a way that, in the first experiment, starting with a glucose concentration of $1 \mathrm{mM}$ during 24 hours, the extracellular glucose concentration was changed to $5 \mathrm{mM}$ and maintained in that value for the entire next day, and then was returned to $1 \mathrm{mM}$ and so on, as shown in figure 1 . The models taken as controls were those with $5 \mathrm{mM}$ constant extracellular glucose concentration, i.e. without perturbation.

We also used the models proposed by Marin et al. [20] for the glycolytic network of HeLa cells grown under normoxia and hypoxia, both under hyperglycemia and then incubated at glucose $5 \mathrm{mM}$, to evaluate the effect of glucose deprivation on cancer glycolysis robustness under these metabolic conditions. The reactions Oxidative Phosphorylation (OxPhos) and the one of the lactate transporter were added to the models, in order to match the reactions of these models to those of Marin et al. [18]. The flux fixed for the reaction OxPhos in the HeLa - normoxia model was the same as the one in the models of Marin et al. [18]. For the HeLa - hipoxia model, the OxPhos flux fixed was $0.00001 \mathrm{mmol} / \mathrm{min}$. The lactate transporter reaction MCT1 was added to the HeLa - normoxia model. It had the same $\mathrm{V}_{\text {max }}, \mathrm{K}_{\mathrm{Lac}(\mathrm{in})}$, $\mathrm{K}_{\mathrm{Lac}(\mathrm{out})}$ and $\mathrm{K}_{\mathrm{eq}}$ values than Marin et al. [18] for hyperglycemia. For the
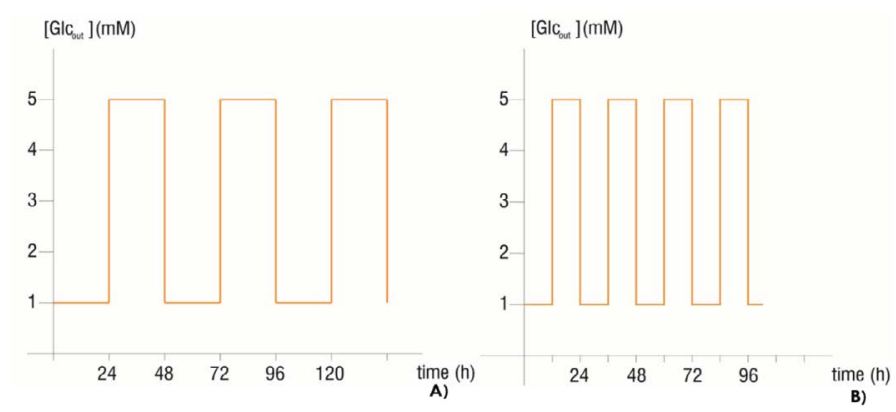

Figure 1. Time serial of extracellular glucose concentration with A) 24 hours' period perturbation and B) 12 hours' period perturbation
HeLa - hypoxia model, the lactate transporter reaction MCT4 added had $\mathrm{K}_{\mathrm{Lac}(\text { in) }}=0.0005$ and $\mathrm{K}_{\mathrm{Lac}(\text { out })}=8.5$. The other reaction parameters had the same values as Marin et al. [18] for hyperglycemia. $\mathrm{K}_{\mathrm{Lac}(\mathrm{in})}$ and $\mathrm{K}_{\mathrm{Lac}(\text { out })}$ are the affinities for intra and extracellular lactate, and $\mathrm{K}_{\mathrm{eq}}$ is the equilibrium constant of the reaction [18].

In order to represent the influence of the glucose deprivation therapy combined with an intracellular acidification treatment on the robustness of cancer glycolysis inside a tumor, all the models mentioned above were subjected to an extracellular glucose reduction from $5 \mathrm{mM}$ to $1 \mathrm{mM}$ and to $\mathrm{pHi}$ changes from 7.8 to 6.4. The former value represents an extreme $p H i$ of cancer cells [15] and the latter one, the $p H i$ after a cellular acidification therapy.

The entropy production rate was calculated using the glycolysis network model of HeLa cell lines at the steady state. The parameters and concentration values used were obtained by modeling the metabolic network for the different metabolic conditions mentioned above.

The modeling of the metabolic network was made in the biochemical network simulator COPASI v 4.16 (http://www.copasi.org).

\section{Thermodynamic framework}

As we have shown in previous works [21], the entropy production rate in cells due to chemical processes driven by the affinity $\mathrm{A}$ is calculated as:

$$
\dot{S}_{i}=\frac{1}{T} \mathrm{~A} \dot{\xi}=-\frac{1}{T} \Delta G \dot{\xi}
$$

Where $T$ is the Temperature and $\dot{\xi}$ is the reaction rate. This value was obtained from COPASI simulation for each one of the reactions.

The variation of free energy of reaction $\left(\Delta G_{k}\right)$ was calculated by the isotherm of reaction.

$$
\Delta G_{k}=\Delta G_{k}^{\oplus}+R T \sum_{i=1}^{n} v_{n} \ln C_{n}
$$

Where $v_{n}$ represent the stoichiometric coefficients, $C_{n}$ concentrations of the involved biomolecules in the stationary state and $\Delta \mathrm{G}_{\mathrm{k}}^{\oplus}$ the standard Gibbs free energy, which was adjusted for the physiological conditions: temperature $T=310.15 \mathrm{~K}$, ionic force $I=0.18 M$ and $p H=7$ [22].

To calculate the rectified standard Gibbs free energy $\left(\Delta \mathrm{G}_{\mathrm{k}}^{\oplus}\right)$ the equation (3) was used.

$$
\begin{aligned}
& \Delta \mathrm{G}_{\mathrm{k}(\mathrm{T}, \mathrm{pH}, \mathrm{I})}^{\oplus}=\sum_{\mathrm{n}} \Delta \mathrm{G}_{\mathrm{n}(\mathrm{T}, \mathrm{pH}, \mathrm{I})}^{\oplus} \\
& \Delta \mathrm{G}_{\mathrm{n}(\mathrm{T}, \mathrm{H}, \mathrm{I}, \mathrm{l}}^{\oplus}=\frac{\mathrm{T}}{298.15} \Delta \mathrm{G}_{\mathrm{n}}^{\theta}+\left(1-\frac{\mathrm{T}}{298.15}\right) \Delta \mathrm{H}^{\theta}+\mathrm{N}_{\mathrm{H}} \mathrm{RT} \ln 10 \cdot \mathrm{pH}-\frac{\mathrm{RT} \alpha\left(\mathrm{z}^{2}-\mathrm{N}_{\mathrm{H}}\right) \sqrt{\mathrm{I}}}{(1+1,6 \sqrt{\mathrm{I}})} ;
\end{aligned}
$$

where: $\alpha$ is the Debye-Hückel constant $\alpha=1.20078\left(\frac{\mathrm{kg}}{\mathrm{mol}}\right)^{1 / 2}, \mathrm{Z}$ is the specie charge, $\mathrm{R}$ is the universal gases constant $8.31 \frac{j}{m o l . k}$ and $\mathrm{N}_{\mathrm{H}}$ is the average number of hydrogen atoms bond to the specie.

The total entropy production rate $\dot{S}_{i(T)}$ for a reaction network is obtained as:

$$
\dot{S}_{i(T)}=\sum_{n} \dot{S}_{i}
$$

where $m$ is the reaction numbers of the network.

\section{Results}

Figure 2 shows the total entropy production rate $\dot{S}_{i(T)}$ values of the HeLa glycolytic network models for hypoglycemia, normoglycemia 
and hyperglycemia; all perturbed with the Heaviside Step Function to simulate an intermittent fasting treatment switching the extracellular glucose concentration from $1 \mathrm{mM}$ to $5 \mathrm{mM}$ with periods of 24 and 12 hours. The 24 hours' period perturbation caused a higher decrease of the $\dot{S}_{i(T)}$ for the three phenotypes, compared with the 12 hours' period perturbation.

Also, the hypoglycemic phenotype cells, which with a constant extracellular glucose concentration showed the highest $\dot{S}_{i(T)}$, were the worst affected with the 24 hours' intermittent fasting, in such a way that the $\dot{S}_{i(T)}$ of the perturbed hypoglycemic phenotype was lower than the $\dot{S}_{i(T)}$ of the normoglycemic phenotype without perturbation.

The figure 3 shows the total entropy production rate values of the glycolytic network for HeLa cells grown under normoxia and hypoxia, and the diminution of the $\dot{S}_{i(T)}$ caused by glucose deprivation. For normal glucose conditions ( $5 \mathrm{mM} \mathrm{Glc}_{\text {out }}$ ), the $\dot{S}_{i(T)}$ of the glycolytic network of the cells grown under hypoxia was higher than those grown under normoxia. Besides, the extracellular glucose deprivation (from $5 \mathrm{mM}$ to $1 \mathrm{mM}$ ) had a greater effect on hypoxic cells because the $\dot{S}_{i(T)}$ decrease was more noticeable for them than for the normoxic cells.

The figure 4 shows the total entropy production rate according to the intracellular $\mathrm{pH}$ for the hypoglycemic, normoglycemic, hypoxic and normoxic phenotypes. It was found a linear relationship between the $\dot{S}_{i(T)}$ and the $\mathrm{pH}$ inside the cell, which translates in as higher the $p H i$, higher the $\dot{S}_{i(T)}$ of cancer glycolysis. Independently of the metabolic condition, the intracellular acidification and reduction of the extracellular glucose concentration led together to a $\dot{S}_{i(T)}$ diminution of the glycolytic network.

\section{Discussion}

\section{Intermittent Fasting}

The intermittent fasting treatment with the 24 hours' period perturbation was more effective than the 12 hours' one, because it achieved a higher diminution of cancer glycolysis robustness $\left(\dot{S}_{i(T)}\right)$ than the 12 hours' treatment for the hypoglycemic, normoglycemic, and hyperglycemic phenotype.

Although Yun et al. [23] discussed the irreversibility of the phenotypic changes induced by glucose deprivation, even after recovering hyperglycemic conditions; here we found that the robustness of the 24 hours perturbed hypoglycemic cells was lower than the robustness of the non-perturbed normoglycemic ones. This result validates the effectiveness of the intermittent fasting as a metabolic cancer therapy.

Several studies had reported the benefits of intermittent fasting as a fighting cancer weapon. Sandor [24] found a linear dose-response relationship between total hours of fasting and the percent of control tumor mass, concluding that intermittent starvation resulted in a significant reduction in Ehrlich ascites tumor growth. Lee et al. [7] showed that cycles of fasting can delay the growth of some cancer

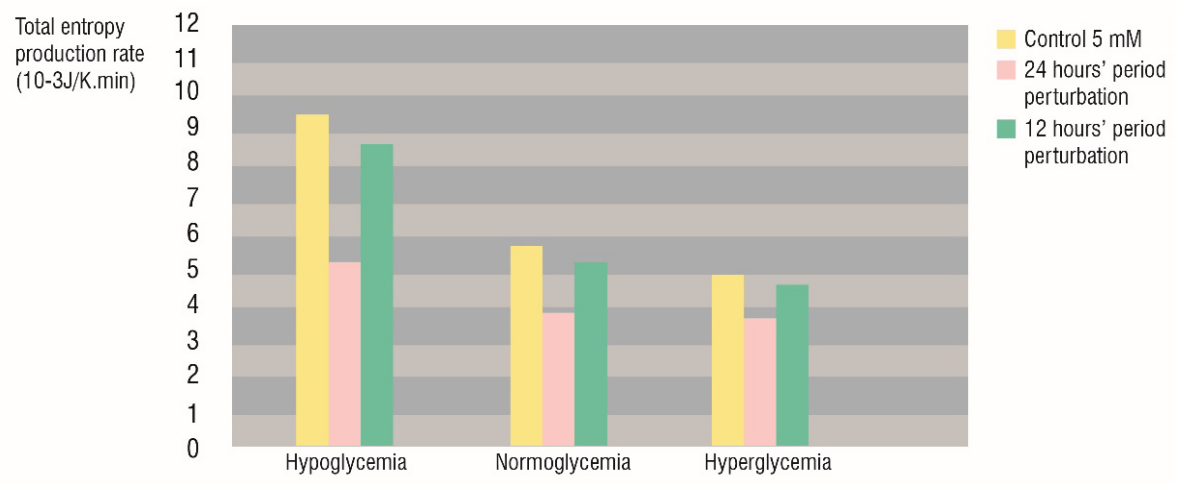

Figure 2. Total entropy production rate of the glycolytic network for HeLa cells grown under hypoglycemia, normoglycemia and hyperglycemia. Yellow: control Glc $=5$ mM, Pink: 24 hours' period $\mathrm{Glc}_{\text {out }}$ perturbation from 1 to $5 \mathrm{mM}$. Green: 12 hours' period $\mathrm{Glc}_{\text {out }}$ perturbation from 1 to $5 \mathrm{mM}$.

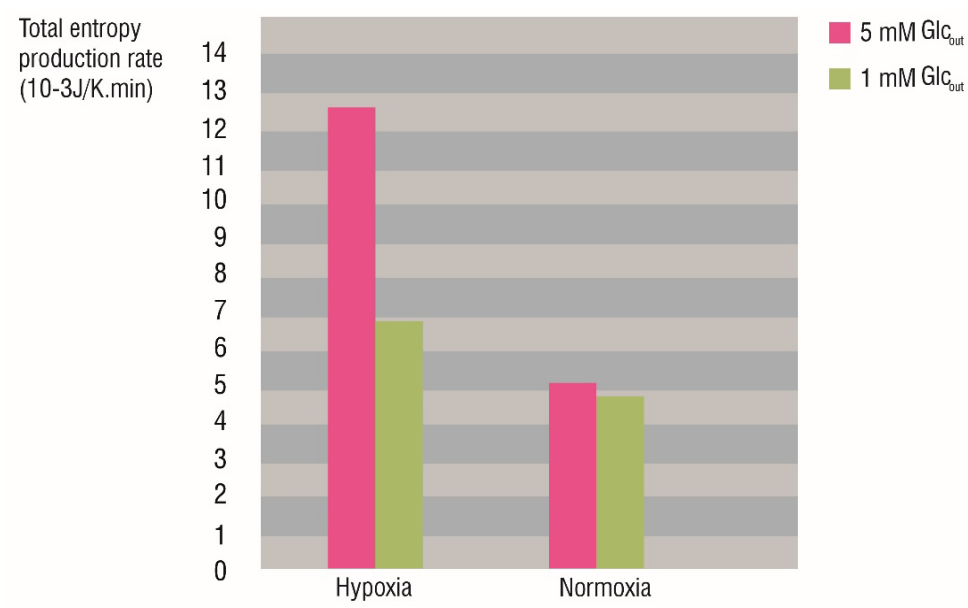

Figure 3. Total entropy production rate of the glycolytic network for HeLa cells grown under hypoxia and normoxia before and after glucose deprivation. Pink: Glc ${ }_{\text {out }}=5$ mM. Green: Glc ${ }_{\text {out }}$ $=1 \mathrm{mM}$. 

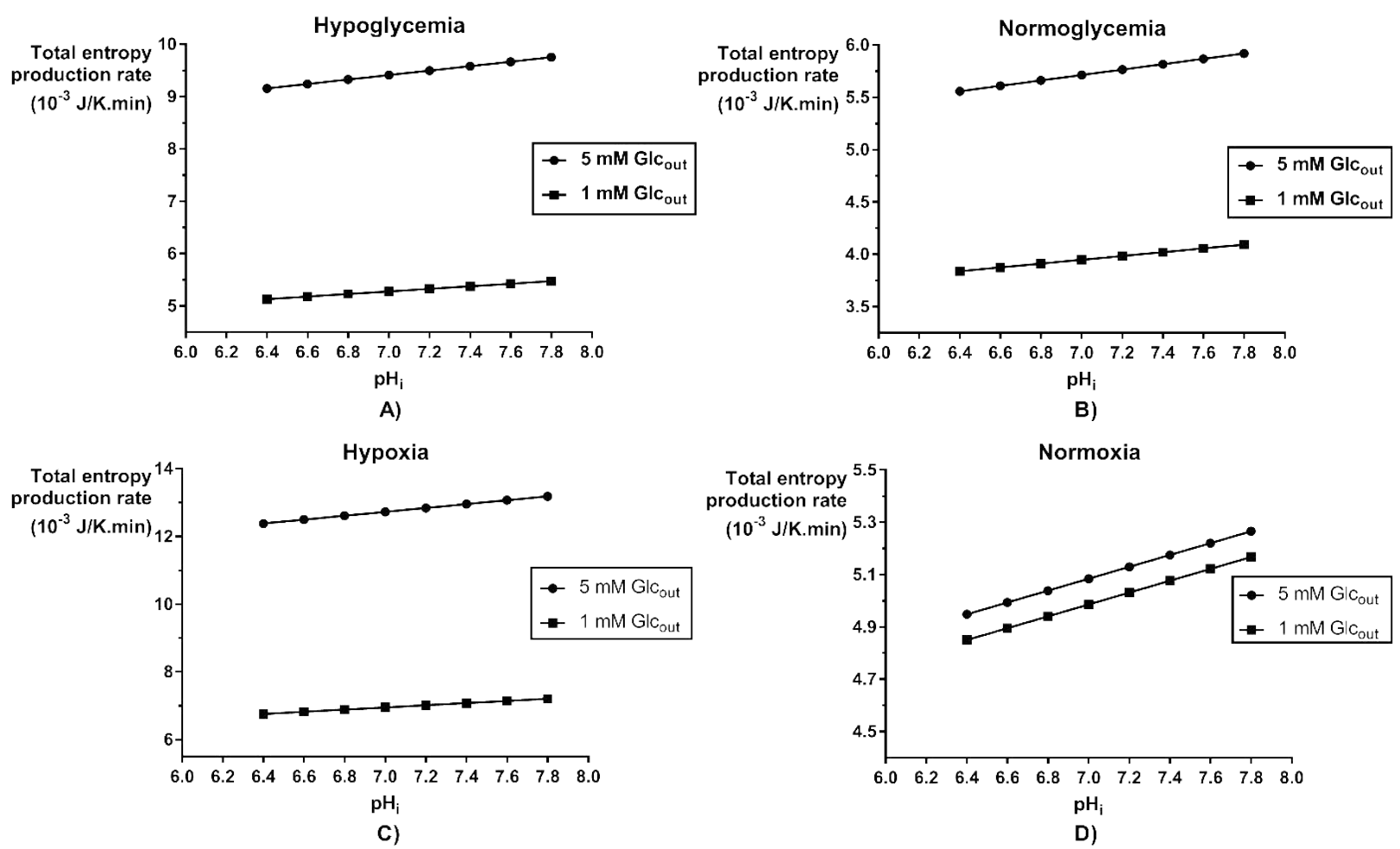

Figure 4. Variation of the total entropy production rate of the glycolytic network for HeLa cells grown under different metabolic conditions, according to intracellular pH values. Cells grown under A) hypoglycemia, B) normoglycemia, C) hypoxia and D) normoxia.

cell types, in some cases as effectively as chemotherapy drugs, but the combination of fasting and chemotherapy cycles provides a more effective, consistent, and potent effect on a wide range of tumors.

\section{Glucose deprivation for cells under hypoxia and normoxia}

For the case of the normoxic and hypoxic HeLa cells, the higher robustness of the glycolytic network for hypoxic cells is due to the increased content and activity of all glycolytic proteins, and pathway flux; mediated by the transcription factor HIF-1a (Hypoxia Induced Factor $1 \alpha)[14,20]$.

The glucose deprivation from $5 \mathrm{mM}$ to $1 \mathrm{mM}$ achieved a robustness decrease for the hypoxic phenotype to a robustness state similar to normoxia. On the other hand, the robustness of the glycolytic network for the normoxic cells was almost the same with extracellular glucose concentration of 5 and $1 \mathrm{mM}$. These results suggest that, unlike hypoxic cells, the cells grown under normoxia were not affected with glucose deprivation.

It has been long appreciated that under hypoxic conditions glycolytic rates are enhanced, with a resulting increase in lactate production [12]. Mjiyad et al. [25] concluded that the effects of glucose deprivation are stronger under hypoxia than under normoxia. Marin et al. [26] showed that under hypoglycemic conditions, hypoxia was unable to increase the glycolytic flux. Instead, a severe depression of glycolytic flux was observed, which did not correlate with the higher levels of expressed oncogenes, and the glycolytic controlling protein content.

Montero et al. [14] recommended that to improve cancer therapies, they should be carried out under normoxia condition. The results here obtained indicate that another way to improve the effectiveness of cancer treatments would be the glucose deprivation of the hypoxic tumor regions, in order to decrease the robustness induced by hypoxia, and match it to the robustness of normoxic tumor areas.

\section{Intracellular acidification and glucose deprivation}

The total entropy production rate was also used to evaluate the influence of the intracellular acidification on cancer glycolysis for the different areas inside a tumor: the hypoxic and hypoglycemic area and the normoxic and normoglycemic [11]. The figure 4 shows a linear relationship between the cancer glycolysis robustness and the intracellular $\mathrm{pH}$ for the phenotypes defined by glucose condition (A and $\mathrm{B}$ ) and the phenotypes defined by the oxygen condition ( $\mathrm{C}$ and D). This result validates the effectiveness of intracellular acidification as a cancer therapy, because lower $p H i$ led to lower $\dot{S}_{i(T)}$ which means lower robustness of the cancer glycolysis. Montero et al. [14] found the same behavior for the entropy production rate of the ATPase reaction according with changes in the intracellular $\mathrm{pH}$. Therefore, the intracellular $\mathrm{pH}$ correction may be an alternative or an adjunct to a metabolic treatment for fighting cancer [27].

When intracellular acidification is combined with glucose deprivation, we found that there is a summation of the effects of both therapies. For all the metabolic conditions analyzed, the $\dot{S}_{i(T)}$ decreased with the diminution of the intracellular $\mathrm{pH}$, but the glucose deprivation therapy had the same outcome, in such a way that for each phenotype considered, the lowest robustness found was the one corresponding to the more acidic $\mathrm{pH}$ and Glucose $1 \mathrm{mM}$ (Figure 4).

\section{Conclusions}

In summary, in this paper we have found that: 
1. The intermittent fasting acts like an alternative cancer therapy because it achieves the robustness decrease of cancer glycolysis, independently of the phenotype established by the glucose condition.

2. The glucose deprivation therapy had a deleterious effect in the metabolic states that under normal glucose conditions showed the highest robustness.

3. The combination of the glucose deprivation and the intracellular acidification therapies had synergic effects on the decrease of cancer glycolysis robustness.

\section{Recommendations}

Extend this study to other tumor cell lines.

\section{Acknowledgements}

Prof. Dr. A. Alzola in memoriam. We would like to thank Prof. Dr. Jacques Rieumont for support and encouragement for this research. One of the authors (JMNV) thanks the CEIICH and the Institute of Physics of the UNAM, Mexico for the warm hospitality and the financial support by DGAPA/DF A/2210/2017. Finally, we thank the anonymous reviewers for their helpful comments and interesting suggestions.

\section{References}

1. World Health Organization (2018) [ http://www.who.int/mediacentre/factsheets/ fs297/en/]

2. Montero S, Durán I, Pomuceno JP, Martín RR, Mesa MD, et al. (2017). How much Damage can make the Glucose in Cancer? J Tumor Res 3: 116.

3. Hanahan D, Weinberg RA (2011) Hallmarks of cancer: the next generation. Cell 144: 646-674. [Crossref]

4. Lee C, Safdie FM, Raffaghello L, Wei M, Madia F, et al. (2011). Reduced IGF-I differentially protects normal and cancer cells and improves chemotherapeutic index in mice. NIH Public Access. Cancer Res 70: 1564-1572. [Crossref]

5. Danhier P, Banki P, Payen VL, Grasso D, Ippolito L, et al. (2017) Cancer metabolism in space and time: Beyond the Warburg effect. Biochim Biophys Acta 1858: 556-572. [Crossref]

6. Gatenby RA, Gillies RJ (2007) Glycolysis in cancer: A potential target for therapy. Int J Biochem Cell Biol 39: 1358-1366. [Crossref]

7. Lee C, Raffaghello L, Brandhorst S, Safdie FM, Bianchi G, et al. (2012). Fasting Cycles Retard Growth of Tumors and Sensitize a Range of Cancer Cell Types to Chemotherapy. Sci Transl Med 4: 124ra27. [Crossref]

8. Buono R, Longo VD (2018) Starvation, Stress Resistance, and Cancer. Trends Endocrinol Metab 29: 271-280. [Crossref]

9. Raffaghello L, Lee C, Safdie FM, Wei M, Madia F, et al. (2008) Starvation-dependent differential stress resistance protects normal but not cancer cells against high-dose chemotherapy. Proc Natl Acad Sci U S A 105: 8215-8220. [Crossref]
10. Eales KL, Hollinshead KE, Tennant DA (2016) Hypoxia and metabolic adaptation of cancer cells. Oncogenesis 5: e190. [Crossref]

11. Allen E, Miéville P, Warren CM, Saghafinia S, Li L, et al. (2016). Metabolic Symbiosis Enables Adaptive Resistance to Anti-angiogenic Therapy that Is Dependent on mTOR Signaling. Cell Rep 15: 1144-1160. [Crossref]

12. Nakazawa MS, Keith B, Simon MC (2016) Oxygen availability and metabolic adaptations. Nat Rev Cancer 16: 663-673. [Crossref]

13. Liou GY, Storz P (2010) Reactive oxygen species in cancer. Free Radic Res 44: 479496. [Crossref]

14. Montero S, Martn RR, Guerra A, Casanella O, Cocho G, et al. (2016) Cancer Glycolysis I: Entropy Production and Sensitvity Analysis in Statonary State. J Adenocarcinoma 1: 1.

15. White KA, Grillo-Hill BK, Barber DL (2017) Cancer cell behaviors mediated by dysregulated $\mathrm{pH}$ dynamics at a glance. $J$ Cell Sci 130: 663-669. [Crossref]

16. Izquierdo-Kulich E, Nieto-Villar JM (2013) Morphogenesis and complexity of the tumor patterns, In: Without Bounds: A Scientifc Canvas of Nonlinearity and Complex Dynamics. Springer, Berlin, Heidelberg.

17. Betancourt-Mar J.A. et al. (2017) Phase transitions in tumor growth: IV relationship between metabolic rate and fractal dimension of human tumor cells. Physica A: 473 344-351.

18. Marín-Hernández A, López-Ramírez SY, Del Mazo-Monsalvo I, Gallardo-Pérez JC, Rodríguez-Enríquez S, et al. (2014) Modeling cancer glycolysis under hypoglycemia, and the role played by the differential expression of glycolytic isoforms. FEBS J 281: 3325-3345. [Crossref]

19. Wolfroam Math World (2018). [http://mathworld.wolfram.com/HeavisideStepFunction html]

20. Marín-Hernández A, Gallardo-pérez JC, Rodríguez-enríquez S, Encalada R, Morenosánchez R, et al. (2011) Modeling cancer glycolysis. Biochimica et Biophysica Acta 1807: 755-767. [Crossref]

21. Izquierdo-Kulich E, Alonso-Becerra E, Nieto-Villar JM (2011) Entropy production rate for avascular tumor growth. Journal of Modern Physics 2: 615

22. Alberty RA(2006) Biochemical thermodynamics: applications of Mathematica. Methods Biochem Anal 48: 1-458. [Crossref]

23. Yun J, Rago C, Cheong I, Pagliarini R, Angenendt P, et al. (2009) Glucose Deprivation Contributes to the Development of KRAS Pathway Mutations in Tumor Cells. Science 325: 1555-1559. [Crossref]

24. Sandor RS (1976) Effects of fasting on growth and glycolysis of the Ehrlich ascites tumor. J Natl Cancer Inst 56: 427-428. [Crossref]

25. El Mjiyad N, Caro-Maldonado A, Ramírez-Peinado S, Muñoz-Pinedo C (2011) Sugarfree approaches to cancer cell killing. Oncogene 30: 253-264. [Crossref]

26. Marín-Hernández A, Gallardo-Pérez JC, Hernández-Reséndiz I, Del Mazo-Monsalvo I, Robledo-Cadena DX, et al. (2016) Hypoglycemia Enhances Epithelial-Mesenchymal Transition and Invasiveness, and Restrains the Warburg Phenotype, in Hypoxic HeLa Cell Cultures and Microspheroids. J Cell Physiol 232: 1346-1359. [Crossref]

27. Schwartz L, Seyfried T, Alfarouk KO, Da Veiga Moreira J, Fais S (2017) Out of Warburg effect: An effective cancer treatment targeting the tumor specific metabolism and dysregulated pH. Semin Cancer Biol 43: 134-138. [Crossref]

Copyright: C2018 Durán I. This is an open-access article distributed under the terms of the Creative Commons Attribution License, which permits unrestricted use, distribution, and reproduction in any medium, provided the original author and source are credited. 